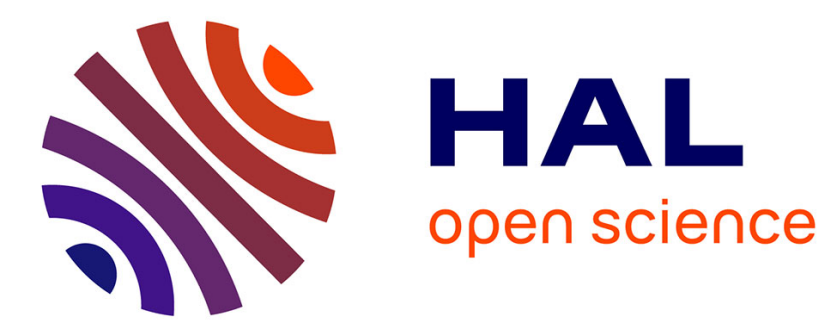

\title{
Unexpected Magnetic Properties of Gas-Stabilized Platinum Nanostructures in the Tunneling Regime
}

Oscar Cespedes, May Wheeler, Timothy Moorsom, Michel Viret

\section{To cite this version:}

Oscar Cespedes, May Wheeler, Timothy Moorsom, Michel Viret. Unexpected Magnetic Properties of Gas-Stabilized Platinum Nanostructures in the Tunneling Regime. Nano Letters, 2015, 15, pp.45 50. $10.1021 / \mathrm{nl} 504254 \mathrm{~d}$. cea-01498274

\section{HAL Id: cea-01498274 https://hal-cea.archives-ouvertes.fr/cea-01498274}

Submitted on 29 Mar 2017

HAL is a multi-disciplinary open access archive for the deposit and dissemination of scientific research documents, whether they are published or not. The documents may come from teaching and research institutions in France or abroad, or from public or private research centers.
L'archive ouverte pluridisciplinaire HAL, est destinée au dépôt et à la diffusion de documents scientifiques de niveau recherche, publiés ou non, émanant des établissements d'enseignement et de recherche français ou étrangers, des laboratoires publics ou privés. 


\title{
Unexpected Magnetic Properties of Gas-Stabilized Platinum Nanostructures in the Tunneling Regime
}

\author{
Oscar Cespedes, ${ }^{* \dagger \dagger}$ May Wheeler, ${ }^{\dagger}$ Timothy Moorsom, $^{\dagger}$ and Michel Viret ${ }^{\ddagger}$ \\ ${ }^{\dagger}$ School of Physics and Astronomy, University of Leeds, Leeds, LS2 9JT, United Kingdom \\ ${ }^{\ddagger}$ Service de Physique de l'Etat Condensé, DSM/IRAMIS/SPEC, CEA Saclay, CNRS URA 2464, F-91191 Gif-Sur-Yvette, France
}

Supporting Information

ABSTRACT: Nanostructured materials often have properties widely different from bulk, imposed by quantum limits to a physical property of the material. This includes, for example, superparamagnetism and quantized conductance, but original properties such as magnetoresistance in nonmagnetic molecular structures may also emerge. In this Letter, we report on the atomic manipulation of platinum nanocontacts in order to induce magnetoresistance. Platinum is a paramagnetic $5 \mathrm{~d}$ metal, but atomic chains of this material have been predicted to be magnetically ordered with a large anisotropy. Remarkably, we

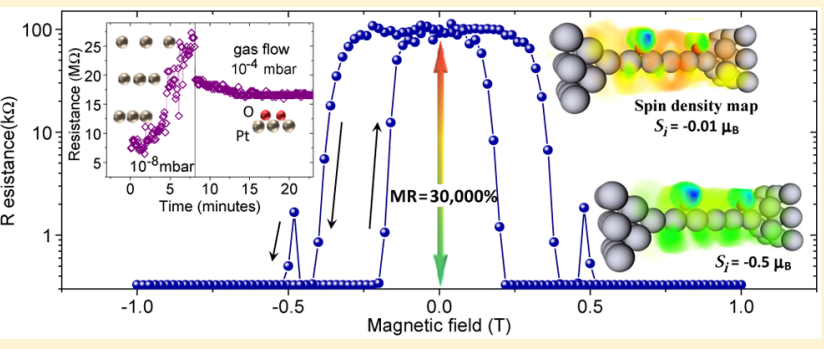
find that a gas flow stabilizes Pt atomic structures in a break junction experiment, where we observe extraordinary resistance changes over $30000 \%$ in a temperature range up to $77 \mathrm{~K}$. Simulations indicate that this behavior may stem from a previously unknown magnetically ordered, low-energy state in platinum oxide atomic chains. This is supported by measurements in Pt/ $\mathrm{PtOx}$ superlattices revealing the presence of a ferromagnetic moment. These properties open new paths of research for atomic scale "dirty" magnetic sensors and quantum devices.

KEYWORDS: Magnetoresistance, platinum oxide, nanocontact, spin-dependent tunneling

\begin{abstract}
alculations suggest that platinum atomic chains of $1 \mathrm{~nm}$
or longer should exhibit spontaneous Hund's rule superparamagnetism at low temperature. ${ }^{1-4}$ This would imply that the spins can be frozen in a direction determined by an external magnetic field. Moreover, the conductance of a single Pt atomic contact is dominated by conducting channels defined by the 8 valence electrons, and it is expected to vary as a function of the chain length and interatomic distance. ${ }^{1,3,5}$ In ideal magnetic atomic structures, only one spin channel may be available, so that the conductance can vary in multiples of $e^{2} / h$ $\left(G_{0} / 2\right)$. Structures with a resistance above $25.9 \mathrm{k} \Omega\left(h / e^{2}\right)$ have little or no orbital overlap, and therefore electrons must tunnel between the atoms forming the chain. In this context, fractional conductance peaks have been observed for $\mathrm{Pd}$ and $\mathrm{Pt}$ atomic contacts and have been attributed to spin polarized transport. ${ }^{6}$ However, these results are not necessarily a proof of magnetic ordering, because the observed conductance peaks around $e^{2} / h$ are rather broad and may have been caused by several suboptimal conducting channels. In fact, fractional conduction peaks have been found in $\mathrm{Pt}$ and $\mathrm{Au}$ wires contaminated by molecules. ${ }^{7,8}$ Shot noise measurements in pure Pt atomic chains have given evidence for a nonmagnetic ground state, but this discrepancy with the theory could be due to spin polarized electrons that do not contribute to the transport. ${ }^{9}$ Molecular structures can show large magnetoresistance (MR) and quantum interference, ${ }^{10-12}$ and a positive $M R$ has been observed at low temperatures in $\mathrm{Pd}$ atomic structures. ${ }^{13}$ Platinum electrodes have also been considered for highly
\end{abstract}

conducting molecular scale junctions. ${ }^{14}$ These highly responsive devices could be thought of as magnetic sensors with potential application in nanoscale memory storage. Furthermore, the possibility of a spin structure where the strength and time scale of the magnetic interactions are controlled at the atomic scale via electromagnetic fields could be the electronic equivalent to optical devices in quantum computing. Manipulation of the contact structure could also result in changes of conductance, Kondo effect, and magnetic ordering. ${ }^{15-17}$

Here, we study atomic contacts of Pt fabricated using the break junction technique. ${ }^{18,19} \mathrm{Pt}$ films for break junctions were deposited using e-beam evaporation from targets containing less than $1 \mathrm{ppm}$ magnetic impurities. The devices were fabricated via lift-off from e-beam patterned PMMA/MMA bilayers on flexible kapton substrates with an overhanging generated via reactive ion etching (see Supporting Information Section 1 for more details). A bridge $20-200 \mathrm{~nm}$ wide is first patterned between two electrodes and the kapton substrate is subsequently folded, breaking and stretching the nanocontact until it forms an atomic or nanoscale chain several atoms long. This technique has been successfully used both in magnetic and superconducting point contacts for spintronics and quantum computing studies. ${ }^{20-22}$ In the case of $\mathrm{Pt}$, it is predicted that as the number of atoms in the chain or the interatomic distance is increased, so is the magnetic moment. ${ }^{1,3}$ The actual

Received: July 9, 2014

Published: December 22, 2014 


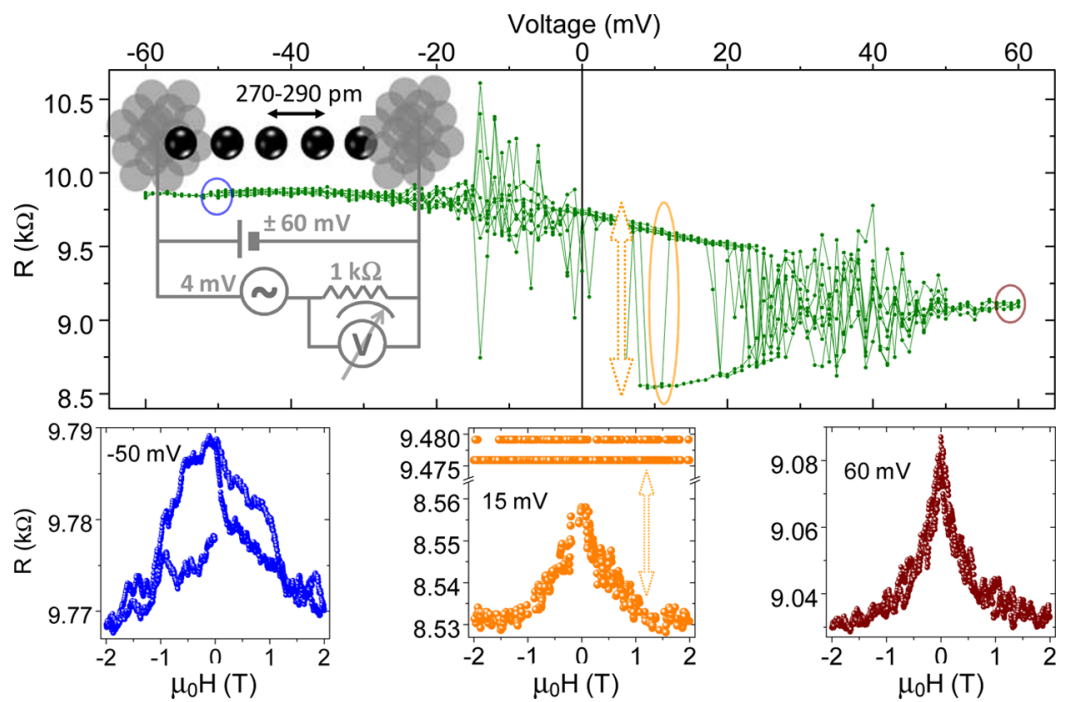

Figure 1. Resistance versus voltage curve and MR for pure Pt atomic chains $\lesssim 1 \mathrm{~nm}$ long formed in high vacuum $\left(10^{-8}\right.$ mbar $)$ and low temperatures $(4.2 \mathrm{~K})$. The resistance is measured using a $4 \mathrm{mV}$ AC voltage. A superimposed dc voltage of up to $\pm 60 \mathrm{mV}$ changes the atomic configuration and/or orbital overlap $(E \sim 10 \mathrm{MV} / \mathrm{m})$. The random telegraph fluctuations are between two metastable states in the $5-25 \mathrm{mV}$ range, one is dependent on magnetic field but the other is not. The schematic shows the measurement setup and a possible chain configuration according to the calculations in ref 1 .
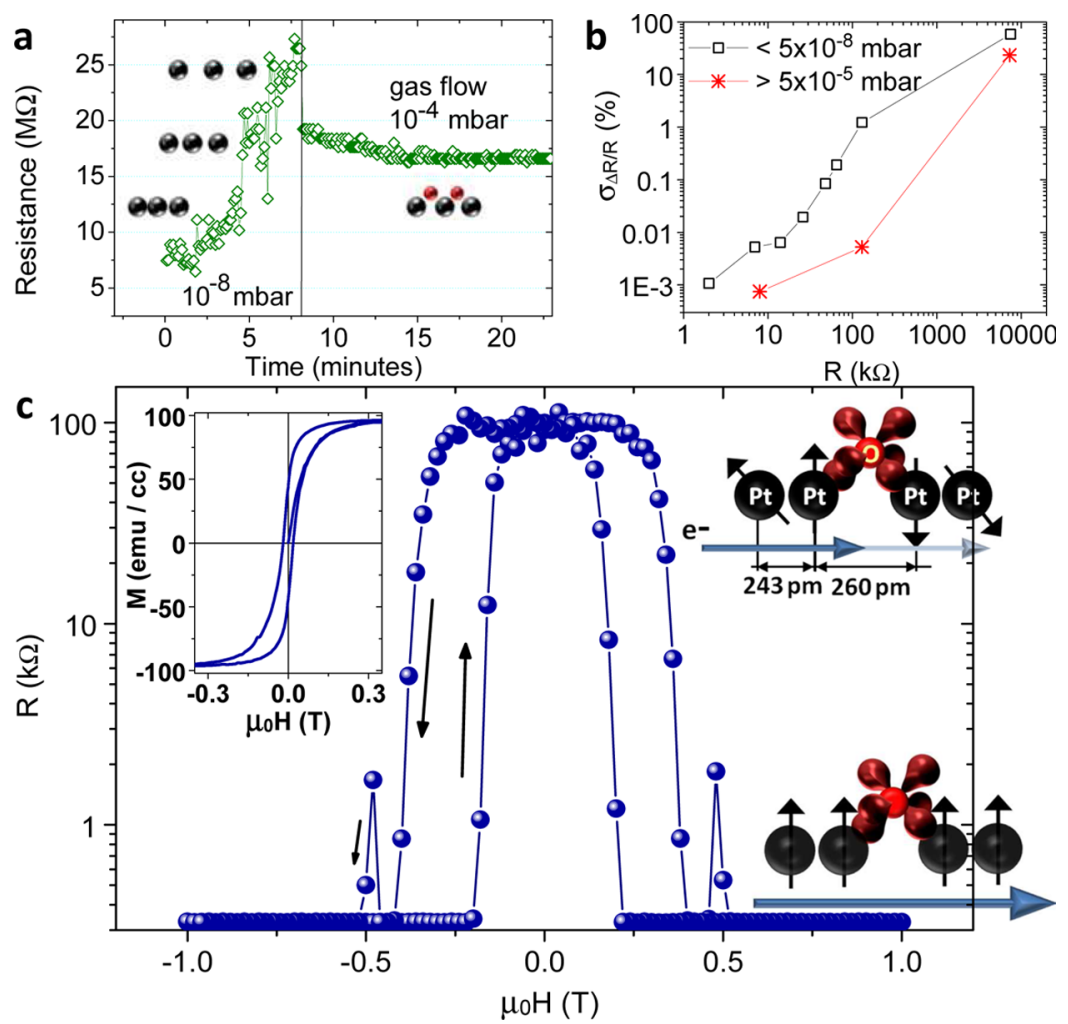

Figure 2. (a) Resistance as a function of time in a Pt atomic chain measured in high and low vacuum. At high vacuum, the contact is breaking, going from 7 to $25 \mathrm{M} \Omega$ in $8 \mathrm{~min}$. An air flow allows the stabilization of nanowires with $R \sim 16 \mathrm{M} \Omega$. (b) Fluctuations of the resistance of Pt chains formed in high and low vacuum as a function of the resistance absolute value. At $\sim 100 \mathrm{k} \Omega$ the peak to valley electrical noise is $\pm 2 \mathrm{k} \Omega$ in high vacuum contacts compared to $\pm 0.03 \mathrm{k} \Omega$ in low vacuum. (c) MR in a Pt nanostructure formed under low vacuum conditions. The contact can measured for hours and has a MR of over $10000 \%$. The inset graph shows the magnetic moment per surface $\mathrm{Pt}$ atom for a $\left[\mathrm{Pt} / \mathrm{PtO}{ }_{x}\right] \mathrm{x} 5$ multilayer $(10 \mathrm{~nm}$ total thickness; raw data in Supporting Information Figure S3).

conductance of a $\mathrm{Pt}$ atomic wire will depend on the exact orbital overlap, that is, the bond between the atoms, or even the wire length. ${ }^{2,23}$ In addition to the atomic distances, it has been shown that the orbital overlap is also a function of the applied voltage and the chain configuration, linear or zigzag. ${ }^{24,25}$
We have not observed any magnetic field dependent transport in $\mathrm{Pt}$ contacts at room temperature with conductance $G$ in the range from 0.001 to $20 G_{0}$ measured at $10^{-9}-10^{-3}$ mbar. However, at $4 \mathrm{~K}$ and for contacts with $G$ close to $G_{0}(R \sim$ $12900 \Omega$ ) we do measure a magnetic field-dependent transport. 

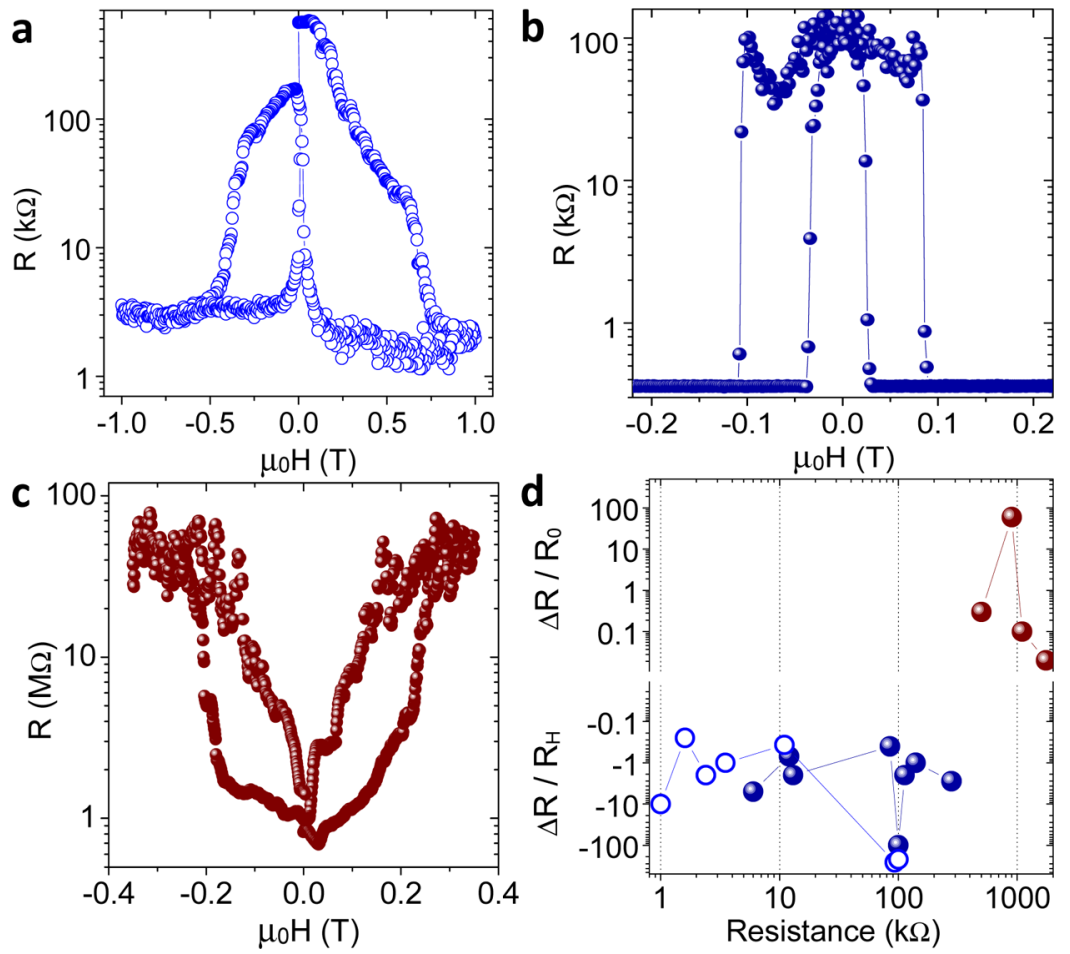

Figure 3. $(\mathrm{a}-\mathrm{c})$ Large MR measured in gas-stabilized Pt nanowires with initial resistance $R \geq 100 \mathrm{k} \Omega$ measured at $4 \mathrm{~K}(\mathrm{a}, \mathrm{c})$ and $77 \mathrm{~K}(\mathrm{~b})$. Data in (c) averaged over $10 \mathrm{mT}$. (d) Variation of the MR as a function of the nanowire resistance measured at $4 \mathrm{~K}$ (open symbols) and $77 \mathrm{~K}$ (filled symbols). The MR turns from negative (reduced resistance in a magnetic field-blue) to positive (increased resistance-red) for structures with resistance above $\sim 300 \mathrm{k} \Omega$.

The MR depends on the applied voltage, as apparent in samples that fluctuate between different resistance values (Figure 1). In these samples, the nanostructure alternates between two similar resistance values at an applied voltage of $15 \mathrm{mV}$. The lower resistance state $(\sim 8.5 \mathrm{k} \Omega)$ shows a MR of $-0.35 \%$, but no MR was measured for the same structure in the higher resistance state at the same voltage $(\sim 9.5 \mathrm{k} \Omega)$. Possible origins for the random telegraph fluctuations between different resistance/magnetic modes are a modified chain and interatomic distance due to atomic displacements, $5,26,27$ voltage-induced instabilities in the orbital overlap, ${ }^{24}$ or electron trapping/detrapping processes. ${ }^{28}$ At voltages of $50-60 \mathrm{mV}$ or below $-20 \mathrm{mV}$, the chain settles in a stable configuration with small fluctuations. Nevertheless, the resistance is different for positive and negative voltages. This could be explained as due to an asymmetric contact leading to different electric field profiles. The wire with the lowest resistance configuration exhibits the highest MR.

Oxygen cannot be ignored in experiments dealing with atomic structures that have not been fabricated and measured in ultrahigh vacuum, as would usually be the case in devices for commercial applications. In $\mathrm{Pt}$, the dissociation of $\mathrm{O}_{2}$ molecules in situ with photons, conduction electrons, or heat leads to two oxygen atoms within two lattice constants of the original molecule with no diffusion of the chemisorbed molecules. ${ }^{29} \mathrm{~A}$ local magnetic moment has been predicted in some amorphous Pt oxides, ${ }^{30}$ so the presence of oxygen molecules need not be an obstacle for Pt nanodevices; it can extend their functionality and strengthen magnetic interactions.

Another benefit is that the presence of gas molecules greatly helps to stabilize the formation of nanostructures with a resistance in the tunneling regime, ${ }^{31}$ where the distance between atoms and therefore the magnetic moments will be larger. ${ }^{3}$ Achieving stable nanocontacts with large interatomic distances may be difficult due to vibrations, applied voltages, thermal fluctuations, and so forth. A dependence of the Fano factor with the conductivity in $\mathrm{Pt}$ atomic chains, that is, an increased variation of the charge fluctuations with lower conductivities has previously been observed. ${ }^{9,32}$ Nevertheless, stable configurations with $G<G_{0}$ can be found in $\mathrm{Pt}$ nanocontacts connected by gas molecules. ${ }^{14}$ Typical lifetimes for single atom and molecular contacts in break junctions are of the order of $1-100 \mathrm{~s} .{ }^{31,33} \mathrm{We}$ find that junctions formed in high vacuum $\left(10^{-9}-10^{-8} \mathrm{mbar}\right)$ with resistances of $0.1-100 \mathrm{M} \Omega$ tend to break after a few minutes at most, as shown in Figure $2 \mathrm{a}$. The transport at high resistances is also unstable with noise fluctuations above $1 \%$ as we exceed $100 \mathrm{k} \Omega$, see Figure $2 \mathrm{~b}$. However, when air is allowed in the chamber to form a relatively low vacuum of $10^{-5}-10^{-3} \mathrm{mbar}$, the contact is stabilized and can be measured for hours at a time with small resistance fluctuations (Figure 2a). Junctions broken in this low vacuum show plateaux at large electrode distances of about 1 $\mathrm{nm}$ with a conductivity of $0.15 \mathrm{G}_{0}(85 \mathrm{k} \Omega)$ that are not observed in the high vacuum breaks; see the Supporting Information.

Although these "dirty" contacts are stable over a wide range of resistances, they are particularly robust at $\sim 100 \mathrm{k} \Omega$, where resistance fluctuations are 3 orders of magnitude smaller than for pure Pt chains formed at high vacuum (Figure $2 b$ ). The MR at temperatures $\leq 77 \mathrm{~K}$ in contacts formed under low vacuum conditions is of up to $-30000 \%$ in fields of the order of 100 $\mathrm{mT}$ (Figure 2c). The shape of the MR curve is also remarkable, as it is reminiscent of a bistable magnetic state system. ${ }^{34,35}$ Before a magnetic field is applied, the resistance for these contacts is of the order of $100 \mathrm{k} \Omega$. However, the low resistance state under applied fields is of $100 \Omega$ to $1 \mathrm{k} \Omega$. This implies that 

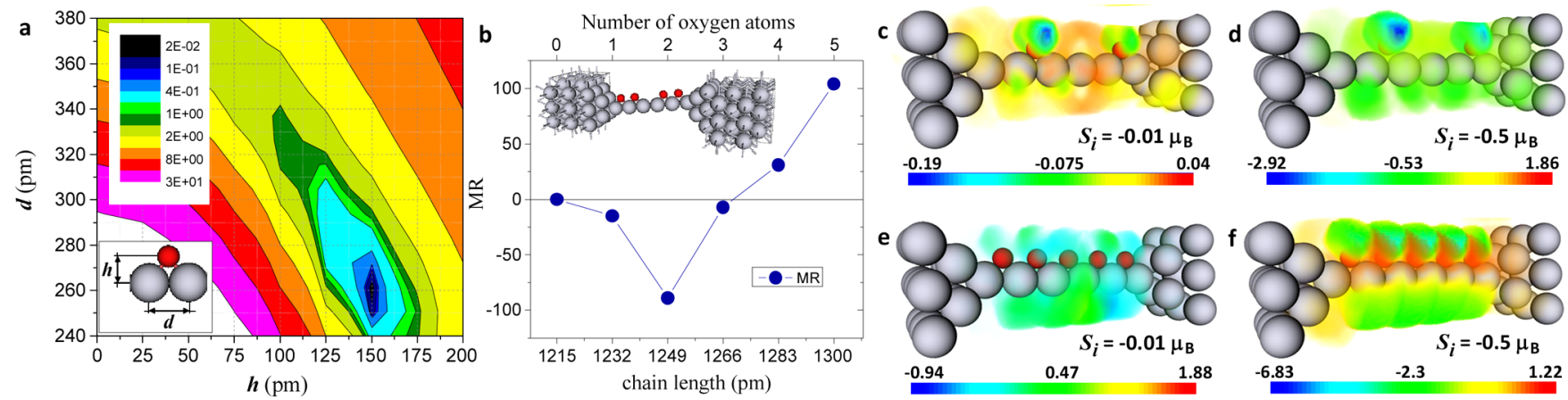

Figure 4. DFT-GGA simulations. (a) Molecular energy of a Pt-O wire (color scale in eV). The ground state is obtained for a Pt-Pt distance $d$ of $260 \mathrm{pm}$ (compared to $243 \mathrm{pm}$ without oxygen). The oxygen atom is at $h=150 \mathrm{pm}$ above the Pt-Pt bond. (b) We calculate the MR in an atomic chain 4 atoms long from the current flow for a fixed voltage of $10 \mathrm{mV}$ as a function of the initial structural spin density: from -0.01 to $-0.5 \mu_{\mathrm{B}} /$ atom. The inset shows the structure used for the calculations with 4 oxygen atoms (PtO). The simulated results show a change in the sign of the MR as we see in our experiments. (c-f) Spin polarization densities (units of $\left.0.1 / \AA^{3}\right)$ for wires with two $(c, d)$ or five $(e, f)$ oxygen atoms and an initial spin per Pt atom of $-0.01(\mathrm{c}-\mathrm{e})$ or $-0.5 \mu_{\mathrm{B}}(\mathrm{d}-\mathrm{f})$.

the cross section of these structures must be a few atoms wide. Otherwise, the conductance quantization would result in higher resistance; even with all spin channels open the resistance of a Pt single atom chain cannot be below $3.2 \mathrm{k} \Omega .^{5}$ Furthermore, the saturation field for these measurements is dependent on the measuring time; lower fields are needed in slower measurements (Supporting Information Section 3). This can be attributed to the magnetic viscosity of the nanowires; at temperatures of $77 \mathrm{~K}$ or below, the magnetization is dependent on thermally activated processes and results in spin dynamics comparable to the measurement time. ${ }^{36,37}$

The highest MR ratios are measured at 77 and $4.2 \mathrm{~K}$ in samples with initial resistance at zero field of $\sim 100 \mathrm{k} \Omega$. Other samples in the 1-200 $\mathrm{k} \Omega$ range present as well very large negative MR values of $100-1000 \%$. Differently from other magnetic oxide nanocontacts, ${ }^{38}$ the MR changes sign as the resistance of the Pt nanostructures increases. Chains formed at low vacuum with a resistance over $200 \mathrm{k} \Omega$ have a positive MR of up to $6000 \%$ (Figure 3 ).

The initial high resistance of all these samples points to tunneling rather than ballistic transport, but the huge MR and the sign changes are difficult to explain only in terms of spin polarized transport. It has been hypothesized that for pure $\mathrm{Pt}$ atomic chains, the spin-dependent effects may be quenched due to the low participation of the magnetic electrons in the transport. $^{9}$ By bonding the outer electrons, oxygen may contribute to enhance the contribution of the magnetic electrons to the charge transport. The magnetic ordering may also be favored by the presence of oxygen atoms in the nanostructures as, for example, in oxygen-deficient $\beta-\mathrm{PtO}_{2}{ }^{30}$ We indeed find that oxidized, $2 \mathrm{~nm}$ thick amorphous $\mathrm{Pt}$ thin films grown by sputter deposition can show ferromagnetic behavior with a small but measurable magnetization of up to 95 $\mathrm{emu} / \mathrm{cc}\left(\sim 0.08 \mu_{\mathrm{B}}\right.$ per Pt atom), a remanence of $0.4-0.5 \mathrm{Ms}$ and a coercive field of $20 \mathrm{mT}$ at $2 \mathrm{~K}$; see Figure $2 \mathrm{c}$. These samples were grown by sputter deposition. Samples with five repeats of $2 \mathrm{~nm}$ Pt layers were deposited ( $10 \mathrm{~nm}$ thick in total). Each layer was plasma oxidized at 2 Torr $\mathrm{O}_{2}$ atmosphere $(2 \times$ $10^{-8}$ Torr base) and $30 \mathrm{~mA}$ current. The results were compared to control samples of pure Pt (Supporting Information Section 2).

In order to find a qualitative explanation for the enhanced $M R$ in gas-stabilized contacts and the change in sign of the MR, we have performed density functional theory (DFT) calculations using the package Atomistix ${ }^{39}$ in the revised generalized gradient approximation (revGGA; see Supporting Information). ${ }^{40,41}$ The lowest energy is found for a $\mathrm{Pt}-\mathrm{O}-\mathrm{Pt}$ atomic chain configuration when compared to other Pt-gas compounds. The oxygen atom is placed in between two $\mathrm{Pt}$ atoms, increasing the $\mathrm{Pt}-\mathrm{Pt}$ equilibrium distance from 243 to $260 \mathrm{pm}$ (Figure 4). The atomic chains we simulate have metastable nonmagnetic and magnetic configurations, where the simulations can converge from initial spin values of 0.01 and $0.5 \mu_{\mathrm{B}}$. The magnetic state is on the order of $0.1 \mathrm{eV}$ lower in energy, and the equilibrium $\mathrm{Pt}-\mathrm{O}$ distance is increased from 198 to 218 pm when the Pt atoms are magnetized. Even though both states are relatively close in energy, they have different resistances. We may consider the magnetic field effect as stabilizing the magnetic configuration at finite temperature, which agrees with our experimental observation of two distinct resistance states. The MR may arise then from an enhanced contribution to the transport of spin polarized electrons, ${ }^{9}$ as well as changes in magnetic ordering and orbital overlap with the magnetic field. ${ }^{24}$

Following this approach, we simulate the MR by calculating the electron current in structures with $4 \mathrm{Pt}$ atoms at a fixed voltage of $10 \mathrm{mV}$ at $77 \mathrm{~K}$ for initial spins of $-0.01 \mu_{\mathrm{B}} / \mathrm{Pt}$ (nonmagnetic or disordered state with resistance $\mathrm{R}_{0}$ ) and -0.5 $\mu_{\mathrm{B}} / \mathrm{Pt}$ (magnetic state with resistance $R_{\mathrm{H}}$ ). The dependence of the electron transport calculated this way with the magnetic field agrees qualitatively with our measurements; changes in resistance from low to high spin states can be positive or negative depending on the configuration of the nanocontact. For example, the result is a MR of $-89 \%$ for Pt chains with 2 oxygen atoms. However, if the chain is further pulled apart so that 5 oxygen atoms can fit in the structure, the MR changes sign and becomes 104\%, reproducing the sign change we observe experimentally, albeit for more resistive structures.

Although the simulations predict a magnetic-dependent transport and a change in the sign of the MR, they cannot reproduce the large effects we measure in some junctions. In order to explain the colossal MR ratios, we may need to consider spin orbit coupling (SOC) in larger nanostructures several atoms wide and the possibility of structural transition induced by the magnetic field. Our model does not include SOC, but it does predict a small displacement of the atoms in the magnetically aligned state. It has been demonstrated that a magnetic field can alter the atomic orbitals or structure of an 
atomic chain and drastically alter its resistance due to SOC. ${ }^{17,42,43}$ Furthermore, small Pt clusters have strong $\mathrm{SOC}^{16}$ leading to anisotropic effects in magnetic atoms, ${ }^{44}$ suggesting that the SOC interaction could induce larger structural changes and play an important role in the magnitude of the MR effect.

The potential of extremely large magnetoresistive ratios and the high stability of our gas-formed $\mathrm{Pt}$ junctions include applications in quantum computing to detect the highly localized fields of qubits or other nanostructures. Furthermore, the ability to tune the spin states and the sign of the resistance change in atomic structures via magnetic and electric fields opens paths of research for "dirty" quantum operators that work at ambient pressures. Further research could also be carried out to investigate the use of magnetic fields in the speed of chemical reactions with nanostructured $\mathrm{PtO}_{2}-$ commonly known as Adams's catalyst. ${ }^{45}$

\section{ASSOCIATED CONTENT}

\section{S Supporting Information}

Additional information and figures on fabrication method, magnetometry and magnetoresistive measurements, and DFT simulations. This material is available free of charge via the Internet at http://pubs.acs.org.

\section{AUTHOR INFORMATION}

\section{Corresponding Author}

*E-mail: o.cespedes@leeds.ac.uk.

\section{Funding}

We are grateful to EPSRC for support via the Grant EP/ K00512X/1.

\section{Notes}

The authors declare no competing financial interest.

\section{ACKNOWLEDGMENTS}

We wish to thank Drs. Steven Achilles, Martin Gratland, Javier Ferrer, Alexander Smogunov, Cyrille Barreteau, and Erio Tosatti for advice on the origin of the MR effects and calculation methods.

\section{REFERENCES}

(1) Delin, A.; Tosatti, E. Phys. Rev. B 2003, 68, 144434.

(2) de la Vega, L.; Martin-Rodero, A.; Yeyati, A. L.; Saul, A. Phys. Rev. B 2004, 70, 113107.

(3) Delin, A.; Tosatti, E. Surf. Sci. 2004, 566, 262-267.

(4) Smogunov, A.; Dal Corso, A.; Delin, A.; Weht, R.; Tosatti, E. Nat. Nanotechnol. 2008, 3 (1), 22-25.

(5) Delin, A.; Tosatti, E. J. Phys.: Condens. Matter 2004, 16 (45), 8061-8074.

(6) Rodrigues, V.; Bettini, J.; Silva, P. C.; Ugarte, D. Phys. Rev. Lett. 2003, 91, 9.

(7) Li, C. Z.; He, H. X.; Bogozi, A.; Bunch, J. S.; Tao, N. J. Appl. Phys. Lett. 2000, 76 (10), 1333-1335.

(8) Untiedt, C.; Dekker, D. M. T.; Djukic, D.; van Ruitenbeek, J. M. Phys. Rev. B 2004, 69, 081401.

(9) Kumar, M.; Tal, O.; Smit, R. H. M.; Smogunov, A.; Tosatti, E.; van Ruitenbeek, J. M. Phys. Rev. B 2013, 88, 245431.

(10) Schmaus, S.; Bagrets, A.; Nahas, Y.; Yamada, T. K.; Bork, A.; Bowen, M.; Beaurepaire, E.; Evers, F.; Wulfhekel, W. Nat. Nanotechnol. 2011, 6 (3), 185-189.

(11) Mahato, R. N.; Lulf, H.; Siekman, M. H.; Kersten, S. P.; Bobbert, P. A.; de Jong, M. P.; De Cola, L.; van der Wiel, W. G. Science 2013, 341 (6143), 257-260.
(12) Guedon, C. M.; Valkenier, H.; Markussen, T.; Thygesen, K. S.; Hummelen, J. C.; van der Molen, S. J. Nat. Nanotechnol. 2012, 7 (5), 304-308.

(13) Ienaga, K.; Nakashima, N.; Inagaki, Y.; Tsujii, H.; Kimura, T.; Kawae, T. Appl. Phys. Lett. 2012, 101, 123114.

(14) Tal, O.; Kiguchi, M.; Thijssen, W. H. A.; Djukic, D.; Untiedt, C.; Smit, R. H. M.; van Ruitenbeek, J. M. Phys. Rev. B 2009, 80, 085427.

(15) Szyja, B. M.; Huu Chuong, N.; Kosov, D.; Doltsinis, N. L. J. Mol. Model. 2013, 19 (10), 4173-4180.

(16) Blonski, P.; Dennler, S.; Hafner, J. J. Chem. Phys. 2011, 134, 034107.

(17) Shi, S. F.; Ralph, D. C. Nat. Nanotechnol. 2007, 2 (9), 522-522.

(18) Yanson, A. I.; Bollinger, G. R.; van den Brom, H. E.; Agrait, N.; van Ruitenbeek, J. M. Nature 1998, 395 (6704), 783-785.

(19) Xiang, D.; Jeong, H.; Lee, T.; Mayer, D. Adv. Mater. 2013, 25 (35), 4845-4867.

(20) Egle, S.; Bacca, C.; Pernau, H.-F.; Huefner, M.; Hinzke, D.; Nowak, U.; Scheer, E. Phys. Rev. B 2010, 81 (13), 134402.

(21) Zgirski, M.; Bretheau, L.; Le Masne, Q.; Pothier, H.; Esteve, D.; Urbina, C. Phys. Rev. Lett. 2011, 106, 257003.

(22) Viret, M.; Berger, S.; Gabureac, M.; Ott, F.; Olligs, D.; Petej, I.; Gregg, J. F.; Fermon, C.; Francinet, G.; Le Goff, G. Phys. Rev. B 2002, 66, 220101.

(23) Autes, G.; Barreteau, C.; Spanjaard, D.; Desjonqueres, M.-C. Phys. Rev. B 2008, 77, 155206.

(24) Nielsen, S. K.; Noat, Y.; Brandbyge, M.; Smit, R. H. M.; Hansen, K.; Chen, L. Y.; Yanson, A. I.; Besenbacher, F.; van Ruitenbeek, J. M. Phys.l Rev. B 2003, 67, 241101.

(25) Vardimon, R.; Yelin, T.; Klionsky, M.; Sarkar, S.; Biller, A.; Kronik, L.; Tal, O. Nano Lett. 2014, 14 (6), 2988-2993.

(26) Ralls, K. S.; Ralph, D. C.; Buhrman, R. A. Phys. Rev. B 1993, 47 (16), 10509-10514.

(27) Cespedes, O.; Jan, G.; Viret, M.; Bari, M.; Coey, J. M. D. J. Appl. Phys. 2003, 93 (10), 8433-8435.

(28) Raghavan, N.; Degraeve, R.; Fantini, A.; Goux, L.; Strangio, S.; Govoreanu, B.; Wouters, D. J.; Groeseneken, G.; Jurczak, M. 2013 IEEE Int. Reliab. Phys. Symp. 2013.

(29) García-Suárez, V. M.; Rocha, A. R.; Bailey, S. W.; Lambert, C. J.; Sanvito, S.; Ferrer, J. Phys. Rev. B 2005, 72 (4), 045437.

(30) Yang, Y.; Sugino, O.; Ohno, T. Phys. Rev. B 2012, 85, 035204.

(31) Tsutsui, M.; Taniguchi, M.; Kawai, T. Nano Lett. 2008, 8 (10), 3293-3297.

(32) Kumar, M.; Smit, R. H. M.; van Ruitenbeek, J. M.; Tal, O. 2011 21st Int. Conf. Noise Fluctuations 2011, 376-380.

(33) Tsutsui, M.; Shoji, K.; Taniguchi, M.; Kawai, T. Nano Lett. 2008, 8 (1), 345-349.

(34) Prezioso, M.; Riminucci, A.; Bergenti, I.; Graziosi, P.; Brunel, D.; Dediu, V. A. Adv. Mater. 2011, 23 (11), 1371-1375.

(35) Sokolov, A.; Sabirianov, R.; Sabirianov, I.; Doudin, B. J. Phys.: Condens. Matter 2009, 21, 485303.

(36) George, R. G. Nature 1959, 183 (4656), 245-245.

(37) Sellmyer, D. J.; Zheng, M.; Skomski, R. J. Phys.: Condens. Matter 2001, 13 (25), R433-R460.

(38) Cespedes, O.; Clifford, E.; Coey, J. M. D. J. Appl. Phys. 2005, 97, 064305 .

(39) Brandbyge, M.; Mozos, J. L.; Ordejon, P.; Taylor, J.; Stokbro, K. Phys. Rev. B 2002, 65, 165401.

(40) Perdew, J. P.; Burke, K.; Ernzerhof, M. Phys. Rev. Lett. 1996, 77 (18), 3865-3868.

(41) Zhang, Y. K.; Yang, W. T. Phys. Rev. Lett. 1998, 80 (4), 890890

(42) Viret, M.; Gabureac, M.; Ott, F.; Fermon, C.; Barreteau, C.; Autes, G.; Guirado-Lopez, R. Eur. Phys. J. B 2006, 51 (1), 1-4.

(43) Bolotin, K. I.; Kuemmeth, F.; Pasupathy, A. N.; Ralph, D. C. Nano Lett. 2006, 6 (1), 123-127.

(44) Gambardella, P.; Rusponi, S.; Veronese, M.; Dhesi, S. S.; Grazioli, C.; Dallmeyer, A.; Cabria, I.; Zeller, R.; Dederichs, P. H.; Kern, K.; Carbone, C.; Brune, H. Science 2003, 300 (5622), 11301133. 
(45) Voorhees, V.; Adams, R. J. Am. Chem. Soc. 1922, 44, 13971405. 